\title{
BMJ Open Representation of racial and ethnic minority groups in cohort studies evaluating risk factors for dementia: protocol for a scoping review
}

\author{
Arjun Krishnan (D) , ${ }^{1,2}$ Louise M Waite, ${ }^{3,4}$ Fiona F Stanaway (D) ${ }^{1}$
}

To cite: Krishnan A, Waite LM, Stanaway FF. Representation of racial and ethnic minority groups in cohort studies evaluating risk factors for dementia: protocol for a scoping review. BMJ Open 2021;11:e044404. doi:10.1136/ bmjopen-2020-044404

- Prepublication history and supplemental material for this paper is available online. To view these files, please visit the journal online (http://dx.doi. org/10.1136/bmjopen-2020044404).

Received 02 September 2020 Revised 23 February 2021 Accepted 17 April 2021

Check for updates

(c) Author(s) (or their employer(s)) 2021. Re-use permitted under CC BY-NC. No commercial re-use. See rights and permissions. Published by BMJ.

${ }^{1}$ School of Public Health, University of Sydney, Sydney, New South Wales, Australia ${ }^{2}$ Fiona Stanley Hospital, Murdoch, Western Australia, Australia

${ }^{3}$ Faculty of Medicine and Health, The University of Sydney Concord Clinical School, Sydney, New South Wales, Australia

${ }^{4}$ The University of Sydney Centre for Education and Research on Ageing, Sydney, New South Wales, Australia

Correspondence to

Dr Fiona F Stanaway;

fiona.stanaway@sydney.edu.au

\section{ABSTRACT}

Introduction Available evidence suggests that some racial/ethnic minority populations may be disproportionately burdened by dementia. Cohort studies are an important tool for defining and understanding the causes behind these racial and ethnic inequalities. However, ethnic minority populations may be more likely to be excluded from such research. Therefore, the aim of this study is to systematically investigate and quantify racial and ethnic minority representation in dementia risk factor research.

Methods and analysis The elements of this protocol have been designed in accordance with the relevant sections of the Preferred Reporting Items for Systematic Reviews and Meta-Analysis Protocols which are specifically applicable to scoping review protocols. We will include population-based cohort studies looking at risk factors for dementia incidence in our review and assess the representation of racial and ethnic minority populations in these studies. We will use multiple strategies to identify relevant studies, including a systematic search of the following electronic databases: MEDLINE (Ovid SP), Embase (Ovid SP) and Scopus. Two review authors will independently perform title and abstract screening, fulltext screening and data extraction. Included cohort studies will be evaluated using a comprehensive framework to assess racial/ethnic minority representation. Logistic regression will also be performed to describe associations between cohort study characteristics and outcomes related to racial and ethnic minority representation. Ethics and dissemination Formal ethical approval is not required to conduct this review as no primary data are to be collected. The final results of this scoping review will be disseminated through publication in peer-reviewed journals and conference presentations.

\section{INTRODUCTION}

Dementia represents a burgeoning noncommunicable disease epidemic in the global health setting. The WHO's 'Global action plan on the public health response to dementia: 2017-2025' estimated that the worldwide prevalence of dementia in 2015 was in the range of 47 million, with a projection for this figure to double by 2030 and triple by $2050 .^{1}$
Strengths and limitations of this study

- We will conduct a thorough and systematic search of the literature.

- Our search will not restrict inclusion on the basis of language of publication or geographical location.

- Our protocol presents a comprehensive framework that includes consideration of selection and measurement bias specific to racial/ethnic minority research.

- Due to the large number of publications arising from individual cohort studies, it is possible that we may miss some published information on race/ethnicity if this is not included in the cohort profile or primary publication.

- Given the nature of the review and the breadth of the topic, we will not be performing a meta-analysis of results.

There is currently a wealth of data derived through cohort studies which have extensively characterised risk factors for dementia in many regions of the world including North America, Europe and parts of Asia..$^{2-4}$ However, examination of cohort study design processes in other disease contexts suggests that racial/ethnic minority status is often overlooked in a substantial portion of longitudinal research. ${ }^{5}$ In an examination of North American and European cardiovascular cohort studies, for instance, Ranganathan and Bhopal concluded that there is a marked shortage of data in non-white ethnic minority populations, ${ }^{5}$ raising concerns of inequity in epidemiological research data. Where systematic exclusion of racial and ethnic minorities in clinical trial research is concerned, a complex interplay of various factors has been identified-chief among which is the practice of exclusion on the basis of language proficiency. ${ }^{67}$

To our knowledge, the extent of representation of racial and ethnic minority participants in dementia risk factor research has not been 
examined or well characterised previously. Our objective is thus to develop a protocol for a scoping review that will identify cohort studies of ageing looking at risk factors for dementia and will appraise these studies on the basis of how racial/ethnic minority status has been considered in their sampling, measurement and analytical processes.

\section{Why is it important to do this review?}

As a result of migration trends over the latter half of the past century, most parts of the world-particularly developed countries-now have a racially and ethnically diverse ageing population. ${ }^{8-10}$ This increased diversity of the older population underlies the importance of including racial/ ethnic minority populations in ageing research. Previous research has suggested that the burden of dementia incidence may be disproportionately greater among particular racial and ethnic minority populations, likely as a consequence of socioeconomic determinants. ${ }^{11-15}$ In recognising the significance of these disparities, the 2020 Lancet Commission report on dementia prevention, intervention and care highlighted the importance of addressing sociodemographic risk factors within ethnic minority groups which may be underpinning differences in dementia incidence. ${ }^{15}$ However, older ethnic minority groups are often under-represented in research. ${ }^{1416}$ The reasons underpinning this relate to factors such as language difficulties, a lack of knowledge about research processes and, in some instances, mistrust stemming from the legacies of institutionalised racism in prior research. ${ }^{16-18}$ In light of this, it stands to reason that racial and ethnic minority members are less likely to be included in dementia risk factor research as well.

Adequate inclusion of ethnic minority participants in cohort studies of dementia risk factors is important in order to determine differences in risk factor prevalence and effects that may translate into different disease burdens. In turn, this may point to the need for targeted health interventions that can reduce health inequalities. Assessment of the quality of this work in terms of the potential for bias and the appropriateness of analyses is also essential. Evaluating ethnic minority representation within the current landscape of dementia risk factor research is vital to understanding where there are limitations in the evidence base and identifying where future research can be improved. To our knowledge, the extent of representation of ethnic minority participants in dementia risk factor research has not been examined or well characterised previously.

\section{Objective}

Our aim is to perform a scoping review that will evaluate ethnic minority representation in cohort studies looking at risk factors for dementia. To this end, we will pursue the following research questions which have been adapted from the work of Ranganathan and Bhopal. ${ }^{5}$

1. What proportion of studies reports the race/ethnicity of their participants?
2. What proportion of studies includes members of racial/ethnic minority groups?

3 . Where race or ethnicity has been measured, how appropriate are the methods used to assess the race/ethnicity of study participants?

4. Where the racial or ethnic composition of the study sample is reported, how does this compare to the racial/ethnic composition of the study population?

5 . What proportion of studies takes race or ethnicity into consideration when analysing results?

6 . What proportion of studies produces results that are likely to be of specific relevance to racial/ethnic minority groups?

7. Are data collected from racial/ethnic minority participants in these studies more likely to have issues with selection and/or measurement bias?

8. Are particular study characteristics associated with greater racial or ethnic minority representation?

\section{METHODS AND ANALYSIS}

\section{Protocol design}

This protocol has been designed following preliminary searches and discussion with content experts. It follows the methodological framework of Arksey and O'Malley ${ }^{19}$ and builds on the research questions previously incorporated in Ranganathan and Bhopal's work on ethnic minority representation in cardiovascular research. ${ }^{5}$ The elements of this protocol have been reported with respect to the sections of the Preferred Reporting Items for Systematic Review and Meta-Analysis protocols ${ }^{20}$ which are specifically relevant to scoping review protocols.

\section{Patient and public involvement}

Our scoping review will not involve any members of the public in our methodological processes.

\section{Eligibility criteria}

Our review will include population-based cohort studies of ageing looking at risk factors for dementia incidence. We will not include case-control or cross-sectional studies. We will also exclude trials as their methodologies are considerably different to cohort studies-particularly with regard to recruitment and duration of follow-up-and are oriented primarily toward investigating the efficacy of interventions rather than the impact of risk factors. We will not place any geographical restrictions on the population cohorts in our review as appropriate racial/ethnic minority representation in cohort studies of dementia is a global concern. Cohort studies to be included in our analysis must investigate the association between a risk factor and overall dementia incidence. Assessment of the dementia outcome in studies must also have been based on clinical criteria and we will not include studies that only measure cognitive impairment without a dementia diagnosis. We will also not include studies whose cohorts have been recruited from specific population groups (eg, institutionalised individuals or those with specific medical conditions). We will include both cohort studies that do 
and do not incorporate ethnic minority representation. Our review will not focus explicitly on any single risk factor for dementia incidence.

\section{Search strategy}

We will search the following electronic databases:

- MEDLINE (via Ovid SP).

- Embase (via Ovid SP).

- Scopus.

We will repeat our search within each database prior to publication of our review in order to retrieve any new studies that have been published over the intervening period since our initial search was conducted. We will not place any restrictions on the basis of language of publication.

Given the immense volume and heterogeneity of publications examining risk factors for dementia, we will employ multiple strategies to efficiently procure all relevant studies. Our initial search will only collect systematic reviews of risk factors for dementia incidence. We will then retrieve the cohort studies included in these systematic reviews for analysis of racial/ethnic minority representation. In instances where multiple publications have originated from the same cohort study, we will collect information from both the original cohort profile report (or demographic description from cohort study websites if available) as well as any later publications specifically relevant to dementia risk factor research. The search strategies for systematic reviews that will be used for MEDLINE (Ovid), Embase (Ovid) and Scopus have been provided in online supplemental appendix 1 and will be adapted for use in other databases. This will be conducted from inception to the date of the search.

We will also seek to retrieve any additional cohort studies that have been published more recently and have thus not been included in any systematic reviews. To this end, a subsequent search of databases will be performed from the date of the search of the most recent well-conducted systematic review (ie, one that we have deemed to have conducted a comprehensive search) identified by the systematic review search. The search strategies for primary studies to be used for MEDLINE (Ovid), Embase (Ovid) and Scopus are shown in online supplemental appendix 2.

Both sets of search strategies were developed by AK and FFS in consultation with an experienced medical librarian. In addition to database searches, further cohort studies of dementia will be identified through contact with experts in the field, a search of cohort study websites, a search of lists of cohort studies of ageing and neurodegenerative disease that have been published online as well as a search of cohort profiles in the International Journal of Epidemiology.

\section{Data management}

Records retrieved in our systematic search will be imported into EndNote V.X9.3 and then into Covidence for ease of screening. The original cohort studies included in the systematic reviews we identify during the first phase of our search will also be imported to EndNote V.X9.3 and then Covidence for full-text review and analysis. Screening and selection processes, including the selection of systematic reviews in our initial search and cohort studies retrieved in our subsequent search, as well as data extraction, will be carried out in Covidence.

\section{Selection process}

Two reviewers (AK and FFS) will independently screen potentially eligible studies for inclusion in our review by screening titles and abstracts yielded by our comprehensive search. We will retrieve full-text articles for all studies that at least one of the review authors identifies as suitable for potential inclusion in the review. Studies will then be selected on the basis of reviewing full-text articles. In cases of disagreement, there will be a consensus meeting between reviewers during which discrepancies will be resolved through discussion with involvement of a third reviewer (LMW) where needed.

\section{Data extraction}

Data will be independently extracted by two reviewers (AK and FFS) using two standardised forms: the first for recording information from retrieved systematic reviews and the second for recording information from the individual cohort studies. These forms will be pilot tested by the two reviewers prior to commencing data extraction. Details of information to be extracted from systematic reviews and individual cohort studies are provided in tables 1 and 2, respectively. On completion of data extraction, both reviewers will compare extracted data in Covidence to identify any errors or disagreements. Disagreements will be resolved through discussion. The finalised data will then be exported from Covidence.

\section{Assessment of racial and ethnic minority representation}

Our assessment of racial and ethnic minority representation within included studies will follow the framework shown in figure 1 . The question components we have included in our framework are divided into three stages and encompass some of the key methodological issues in epidemiological research where racial and ethnic minority representation is concerned. The stages of our framework are elucidated below.

\section{Stage 1}

The foremost consideration within our framework is whether race or ethnicity has been discussed as part of

Table 1 Data extraction items for systematic reviews identified in the first search phase

\begin{tabular}{ll}
\hline Study details & Citation \\
Study results & Risk factor(s) being examined \\
& Citations for all cohort studies included in \\
& the review that meet the inclusion criteria \\
& Date of literature search \\
& Comprehensive search (yes/no)
\end{tabular}


Table 2 Data extraction items for cohort studies identified from either the first or second phase of our search

\begin{tabular}{|c|c|}
\hline Study details & $\begin{array}{l}\text { Citation } \\
\text { Study design } \\
\text { Risk factor(s)/research question being examined } \\
\text { Specific outcome(s) being assessed } \\
\text { Study location and setting } \\
\text { Total duration of study (including follow-up period) }\end{array}$ \\
\hline Study participants & $\begin{array}{l}\text { Study population described } \\
\text { Race/ethnicity of participants reported (yes/no) } \\
\text { Specific ethnic minority populations described } \\
\text { Racial/ethnic minority populations actively excluded (yes/no) and the reasons used } \\
\text { Number of participants by race/ethnicity (with comparison with ethnicity of study population likely from } \\
\text { national data but also from the geographical area of the study where possible) } \\
\text { Mean age of participants } \\
\text { Sex of participants } \\
\text { Losses to follow-up (by ethnicity if data available) }\end{array}$ \\
\hline Study methods and outcomes & $\begin{array}{l}\text { Method of ascertainment of race/ethnicity (if applicable) } \\
\text { Details of racial/ethnic minority status as a consideration in data analysis (including any aggregation of } \\
\text { populations and sample sizes) } \\
\text { Method of risk factor and dementia assessment (by race/ethnicity if data available; including assessment } \\
\text { of risk of bias of risk factor and dementia assessment in different racial and ethnic groups) } \\
\text { Any results deemed specifically relevant to racial/ethnic minority populations } \\
\text { - Evidence of risk factor to outcome relationship modification by race/ethnicity } \\
\text { - Evidence of ethnicity as a risk factor and/or difference in prevalence of identified risk factor(s) by race/ } \\
\text { ethnicity }\end{array}$ \\
\hline
\end{tabular}

the study's selection process or its description of participant characteristics. This may include, but is not limited to, race/ethnicity being described within the participant inclusion/exclusion criteria or as part of the results section where the participants in the study cohort are described in some detail.

\section{Stage 2}

We define racial/ethnic minorities as groups who are numerically smaller and possess ethnic, religious or linguistic characteristics that are different from those in the rest of the population. ${ }^{21}$ Active exclusion of racial/ ethnic minority participants will be considered if there are explicit inclusion/exclusion criteria in the cohort study that disadvantage or disqualify participants based on race/ethnicity or its related factors. This may include, but once again is not limited to, restrictions placed on the basis of language proficiency, citizenship or country of birth.

Evaluating underinclusion of racial and ethnic minority participants in studies is somewhat more nuanced and, in a broad sense, comprises two situations. First, underinclusion may relate to the study's racial/ethnic composition not being representative of the population from which participants are recruited.

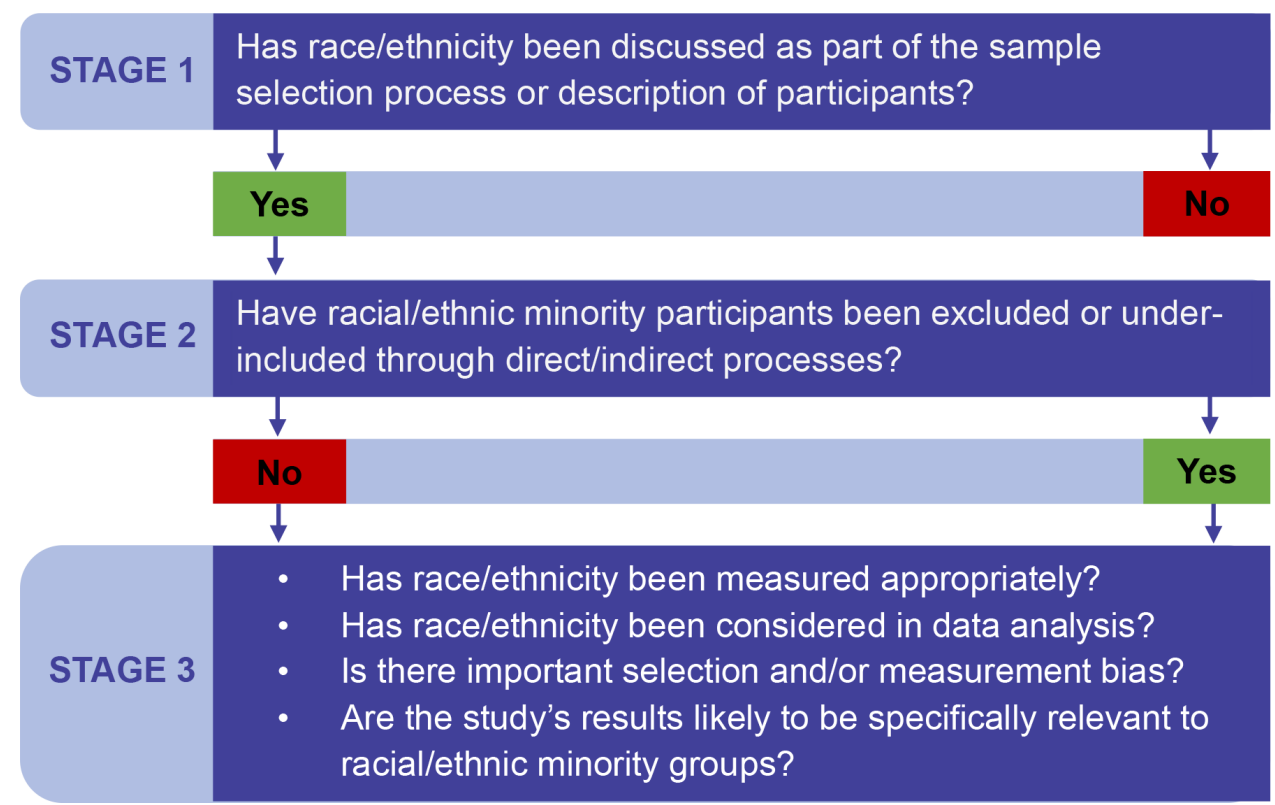

Figure 1 Framework for evaluation of racial/ethnic minority representation within included cohort studies. 
Alternatively, underinclusion may also relate to instances where although the racial/ethnic composition of the study sample is reflective of the geographical area from which it is drawn, the study sample may not necessarily be reflective of the country's ethnic composition as a whole. This may be the case for studies which are primarily conducted in rural areas where racial/ethnic diversity may be considerably lower than the rest of the country. It may also include a consideration of the timing of the study. For example, studies conducted several decades ago may have been representative of the level of racial/ethnic diversity of the older population at that time in a given setting but would not be representative of current levels of racial/ ethnic diversity.

\section{Stage 3}

\section{Has race/ethnicity been measured appropriately?}

The first aspect of our evaluation within this stage relates to whether or not race/ethnicity has been measured appropriately. Though race and ethnicity are complex and multidimensional concepts that are often difficult to accurately measure, there are several national guidelines and recommended frameworks endorsed by scientific journals that can potentially be adopted in cohort studies. ${ }^{22-25}$ The British Medical Journal $(\mathrm{BMJ})$, in particular, has endorsed a reporting framework for publications in their journal that provides authors guidance as to how ethnicity, race and culture should be measured and reported. ${ }^{24}$ In general terms, this framework and the national guidelines encourage the measurement of ethnicity using multiple variables that are ideally collected through self-report. Bhopal has also published a comprehensive glossary that elucidates, in some detail, appropriate conventions for categorisation of ethnicity within a number of populations throughout the world. ${ }^{26}$ Where relevant, we will evaluate the appropriateness of how ethnicity data have been measured using national guidelines for those studies originating from countries which have published such recommendations. In the instance where no national guidelines have been published for a given setting, we will use the framework endorsed by the BMJ as well as the published glossary by Bhopal as reference points for making our judgements.

\section{Has race or ethnicity been considered in data analysis?}

We will also consider if race or ethnicity has been incorporated into data analysis. For instance, studies may adjust for race/ethnicity as a confounder, they may stratify results by race/ethnicity, they may examine for interactions by race/ethnicity, or they may exclude racial and ethnic minority participants from analyses to reduce heterogeneity in the racial/ethnic majority population. Part of this assessment also requires consideration of the way that racial/ethnic groups are categorised in analyses and how this may impact on the value of the results to specific ethnic groups. For instance, the combining of multiple diverse groups together into a single category may completely remove the ability of the results to provide any useful information.

\section{Is there important selection and/or measurement bias?}

We will evaluate the potential for differences in terms of selection and measurement bias between data collected from racial/ethnic minority and racial/ethnic majority participants. Selection bias, for example, may be problematic when losses to follow-up are disproportionately greater among racial and ethnic minority participants compared with those in the racial/ethnic majority population. It may also occur when language proficiency is used as a study eligibility criterion, as including only those racial/ethnic minority persons with high language proficiency could lead to a nonrepresentative sample. Measurement bias, on the other hand, is likely to manifest when appropriate study materials or data collection processes have not appropriately considered the potential for measurement issues in racial and ethnic minority participants. For instance, if translated and culturally adapted data collection tools or interpreters are not used in those with limited proficiency in the national language, this may mean that measurement of exposure or outcome may have an increased risk of bias in racial and ethnic minority participants with this limited language proficiency. This is likely to be particularly problematic in assessment of dementia status. We will also consider if processes for the diagnosis of dementia-such as the commonly used approach of consensus meetings to establish the diagnosis-were conducted blind to ethnicity.

Are the study's results likely to be specifically relevant to racial and ethnic minority groups?

The final component of this stage in our framework is an amalgamation of several considerations. This includes how appropriately ethnicity has been measured or categorised, if/how social determinants have been factored into the interpretation of observed differences in dementia incidence between racial/ethnic groups, and if the analyses are sufficiently powered to provide meaningful results. It will also consider evidence for differences in the prevalence of different risk factors by race/ethnicity or differences in risk factor-outcome relationships by race/ ethnicity that would have the potential to inform policy and strategies targeting dementia risk in racial and ethnic minority populations.

\section{Data synthesis}

As this is a scoping review and we are not aiming to provide summary estimates of risk factor relationships, there will be no meta-analysis. However, we will collate findings from our assessment of racial and ethnic minority representation within included studies to generate the following:

1. The proportion of studies including participants from racial and ethnic minority groups. 
2. The proportion of studies where race/ethnicity has been measured appropriately_-both with respect to all included cohort studies as well as those studies including racial and ethnic minority participants as denominators.

3. The proportion of studies whose racial or ethnic composition is representative of the geographical regions and/or countries from which the study populations have been derived.

4. The proportion of studies that takes race/ethnicity into consideration when analysing results.

5. The proportion of studies incorporating racial and ethnic minority participants that are unlikely to have an increased risk of selection bias and/or measurement bias in results from racial and ethnic minority participants.

6. The proportion of studies producing results that are likely to be of specific relevance to racial and ethnic minority groups.

These summary measures will also be disaggregated by geographical location as well as the type of country (ie, high/middle/low-income status).

We will also perform logistic regression using SPSS V.26 (IBM Corporation) to examine what characteristics of cohort studies are associated with greater racial and ethnic minority representation as defined by the above six summary measures. The independent variables to be evaluated in our analysis will include: the country in which the cohort study is undertaken, funding source (if available), year of publication, number of years of follow-up as well as the risk factor(s) which are being considered in the study.

\section{ETHICS AND DISSEMINATION}

Ethics approval to conduct this research is not required as primary data will not be collected. The results of our scoping review will be published in a peer-reviewed journal and following publication, we will also develop plain language summaries of our results for wider dissemination to members of the public.

Contributors FFS conceived the idea for the study and guided the research team throughout all stages of protocol development. AK and FFS planned and designed the study protocol with input from LMW regarding appropriate study inclusion criteria. AK prepared the first manuscript draft with all authors contributing critical feedback and advice. All authors have approved and contributed to the final version of the protocol.

Funding This research received no specific grant from any funding agency in the public, commercial or not-for-profit sectors. Open-access fees were covered by the ARC Centre of Excellence in Population Ageing Research (CEPAR).

Competing interests None declared.

Patient consent for publication Not required.

Provenance and peer review Not commissioned; externally peer reviewed.

Supplemental material This content has been supplied by the author(s). It has not been vetted by BMJ Publishing Group Limited (BMJ) and may not have been peer-reviewed. Any opinions or recommendations discussed are solely those of the author(s) and are not endorsed by BMJ. BMJ disclaims all liability and responsibility arising from any reliance placed on the content. Where the content includes any translated material, BMJ does not warrant the accuracy and reliability of the translations (including but not limited to local regulations, clinical guidelines, terminology, drug names and drug dosages), and is not responsible for any error and/or omissions arising from translation and adaptation or otherwise.

Open access This is an open access article distributed in accordance with the Creative Commons Attribution Non Commercial (CC BY-NC 4.0) license, which permits others to distribute, remix, adapt, build upon this work non-commercially, and license their derivative works on different terms, provided the original work is properly cited, appropriate credit is given, any changes made indicated, and the use is non-commercial. See: http://creativecommons.org/licenses/by-nc/4.0/.

ORCID iDs

Arjun Krishnan http://orcid.org/0000-0001-5750-2069

Fiona F Stanaway http://orcid.org/0000-0003-2104-3010

\section{REFERENCES}

1 World Health Organization. Global action plan on the public health response to dementia 2017-2025. Geneva: World Health Organization, 2017.

2 Bellou V, Belbasis L, Tzoulaki I, et al. Systematic evaluation of the associations between environmental risk factors and dementia: an umbrella review of systematic reviews and meta-analyses. Alzheimers Dement 2017;13:406-18.

3 Peters R, Booth A, Rockwood K, et al. Combining modifiable risk factors and risk of dementia: a systematic review and meta-analysis. BMJ Open 2019;9:e022846.

4 Wang X-J, Xu W, Li J-Q, et al. Early-life risk factors for dementia and cognitive impairment in later life: a systematic review and metaanalysis. J Alzheimers Dis 2019;67:221-9.

5 Ranganathan M, Bhopal R. Exclusion and inclusion of nonwhite ethnic minority groups in 72 North American and European cardiovascular cohort studies. PLoS Med 2006;3:e44.

6 Hussain-Gambles M, Atkin K, Leese B. Why ethnic minority groups are under-represented in clinical trials: a review of the literature. Health Soc Care Community 2004;12:382-8.

7 Hughson J-A, Woodward-Kron R, Parker A, et al. A review of approaches to improve participation of culturally and linguistically diverse populations in clinical trials. Trials 2016;17:263.

8 International Organization for Migration. World migration report 2020. Geneva, Switzerland, 2020. Available: https://www.un.org/sites/un2. un.org/files/wmr_2020.pdf [Accessed 13 Jul 2020].

9 Australian Institute of Health and Welfare. Older Australia at a glance. Canberra, 2018. Available: https://www.aihw.gov.au/reports/olderpeople/older-australia-at-a-glance/contents/demographics-of-olderaustralians/australia-s-changing-age-and-gender-profile [Accessed 13 Jul 2020].

10 Ortman JM, Velkoff VA, Hogan $\mathrm{H}$. An aging nation: the older population in the United States. Maryland, United States, 2014. Available: https://www.census.gov/prod/2014pubs/p25-1140.pdf [Accessed 13 Jul 2020].

11 Chen C, Zissimopoulos JM. Racial and ethnic differences in trends in dementia prevalence and risk factors in the United States. Alzheimers Dement 2018;4:510-20.

12 Rodriguez FS, Aranda MP, Lloyd DA, et al. Racial and ethnic disparities in dementia risk among individuals with low education. Am J Geriatr Psychiatry 2018;26:966-76.

13 Yaffe K, Falvey C, Harris TB, et al. Effect of socioeconomic disparities on incidence of dementia among biracial older adults: prospective study. BMJ 2013;347:f7051.

14 Babulal GM, Quiroz YT, Albensi BC, et al. Perspectives on ethnic and racial disparities in Alzheimer's disease and related dementias: update and areas of immediate need. Alzheimers Dement 2019;15:292-312.

15 Livingston G, Huntley J, Sommerlad A, et al. Dementia prevention, intervention, and care: 2020 report of the Lancet Commission. Lancet 2020;396:413-46.

16 Bécares L, Kapadia D, Nazroo J. Neglect of older ethnic minority people in UK research and policy. BMJ 2020;368:m212.

17 Guillemin M, Gillam L, Barnard E, et al. "We're checking them out": Indigenous and non-Indigenous research participants' accounts of deciding to be involved in research. Int J Equity Health 2016;15:8.

18 Williams MM, Scharff DP, Mathews KJ, et al. Barriers and facilitators of African American participation in Alzheimer disease biomarker research. Alzheimer Dis Assoc Disord 2010;24 Suppl:S24-9.

19 Arksey H, O'Malley L. Scoping studies: towards a methodological framework. Int J Soc Res Methodol 2005;8:19-32.

20 Shamseer L, Moher D, Clarke M, et al. Preferred reporting items for systematic review and meta-analysis protocols (PRISMA-P) 2015: elaboration and explanation. BMJ 2015;349:g7647. 
21 United Nations Human Rights OotHC. Minority rights: international standards and guidance for implementation. New York and Geneva, 2010. Available: https://www.ohchr.org/Documents/Publications/ MinorityRights_en.pdf [Accessed 7 Jan 2021].

22 Australian Bureau of Statistics. Australian standard classification of cultural and ethnic groups (ASCCEG), 2019. Australian Bureau of statistics, Australian government, 2019. Available: https://www.abs. gov.au/ausstats/abs@.nsf/mf/1249.0 [Accessed 9 Jul 2020].

23 Office for National Statistics. Ethnic group, national identity and religion, 2016. Available: https://www.ons.gov.uk/methodology/clas sificationsandstandards/measuringequality/ethnicgroupnationalident ityandreligion [Accessed 16 Jul 2020]

24 McKenzie K, Crowcroft NS, race D. Describing race, ethnicity, and culture in medical research. BMJ 1996;312:1054.

25 National Institute of Health. Definitions for NIH diversity programs and for other reporting purposes, 2015. Available: https://grants.nih. gov/grants/guide/notice-files/NOT-OD-15-089.html [Accessed $15 \mathrm{Ju}$ 2020].

26 Bhopal RS. Migration, ethnicity, race, and health in multicultural societies. 2 edn. Oxford: Oxford University Press, 2014. 